\title{
LA DEPRESIÓN POSTPARTO: UN ESTUDIO EXPLORATORIO CON UNA MUESTRA DE MUJERES VALENCIANAS
}

\author{
ELVIRA MARTÍNEZ, MERCEDES TOLEDO, MARÍA PINEDA ${ }^{1}$, JAVIER MONLEÓN ${ }^{1}$, \\ JAVIER FERRERO y M. PILAR BARRETO \\ Facultad de Psicología, Universidad de Valencia \\ ${ }^{1}$ Servicio de Obstetricia. Centro Maternal. Hospital Universitario La Fe, Valencia
}

(Aceptado en noviembre de 2000)

El objetivo del presente estudio era analizar la existencia de depresión postparto (depresión y ansiedad) en una muestra de mujeres de la Comunidad Valenciana así como su evolución dos meses después del parto. La muestra estuvo compuesta por 101 mujeres residentes en la Ciudad de Valencia y pueblos de alrededor que dieron a luz en el Centro Maternal del Hospital Universitario "La Fe" de Valencia. Los instrumentos utilizados fueron los siguientes: La Escala de Edimburgo para la Depresión postparto (EPDS, Cox, Holden y Sagovsky, 1987) el Inventario de Ansiedad Estado-Rasgo (STAI, Spielberger, Gorsuch y Lushene, 1970) y el Inventario de Depresión de Beck (BDI, Beck, Rush, Shaw y Emery, 1979). Los principales resultados indican que un porcentaje numeroso de mujeres supera los puntos de corte establecidos para el diagnóstico de depresión según la EPDS en ambos momentos del estudio: $32,67 \%$ y $26,73 \%$ respectivamente. Las mujeres que habían tenido una experiencia regular o pésima del embarazo y aquellas que se sentían poco capaces para afrontar cambios eran las que presentaban las puntuaciones más elevadas en depresión $(p \leq 0,05)$. Así pues, se observa una prevalencia importante de depresión postparto, destacando también la experiencia del embarazo y la capacidad para afrontar cambios como dos variables relacionadas con la depresión en estas mujeres.

Palabras clave: Evaluación, postparto, depresión, ansiedad.

\section{The postnatal depression: an exploratory study in a sample of Spanish women from the Valencian Community}

A The aim of the present study was to analyse the existence of postnatal depression (anxiety and depression) in a sample of women from the Valencian Community (East of Spain) as well as its evolution two months after delivery. A sample of 101 women resident in the City of Valencia and surrounding villages, that gave birth in the Maternal Ward of the University Hospital "La Fe" of Valencia, were surveyed. Depression was assessed by means of the Edinburgh Postnatal Depression Scale (EPDS; Cox, Holden y Sagovsky, 1987),the State-Trait Anxiety Inventory (STAI; Spielberger, Gorsuch y Lushene, 1970) and the Beck Depression Inventory (BDI; Beck, Rush, Shaw y Emery, 1979). The main results point out that a high percentage of women overcomes the cutoff points established for diagnosis of depression according to EPDS in both moments of the study ( $32 \%$ and $26 \%$ respectively). Depression level was related with bad pregnancy and difficulties of coping with the new experience $(p \leq 0,05)$. An important prevalence of postnatal depression was observed. The ways they live pregnancy and cope with changes have proved to be related to depression in these women.

Key words: Assessment, postnatal depression, anxiety.

Gorrespondencia: Elvira Martínez Besteiro, Departamento de Personalidad, Evaluación y Tratamientos Psicológicos, Facultad de Psicología, Avda. Blasco Ibáñez, 21, 46010 Valencia. Tfno.: 963864 476, Fax: 963864 669, Correo-e: Elvira.Martinez@uv.es
Agradecimientos: Esta investigación se ha podido llevar a cabo gracias al proyecto subvencionado por el Organismo Público Valenciano de Investigación O.P.V.I., dependiente de la Consellería de Cultura, Educación y Ciencia. (Acción Especial de I+D, 1999) 


\section{INTRODUCCIÓN}

El análisis de las alteraciones psicológicas que pueden tener lugar en el postparto no es reciente. Victoroff acuñó el término maternity blues en 1952 (Pritchard y Harris, 1996) y Hamilton (1962, citado en Llavona, Muruaga y Nogueiras, 1986) definió la depresión post-parto como un síndrome caracterizado por un estado depresivo moderado, al que acompañan síntomas como lloros, fatiga, pérdida de energía, incomodidad física, problemas de concentración, pérdida de apetito, etc. cuya duración puede variar desde unas horas hasta unos días. Otro trastorno mental perinatal lo constituyen las "psicosis puerperales» descritas fundamentalmente como psicosis afectivas o funcionales que comienzan dentro de los tres meses tras el parto (Terp y Mortensen, 1998).

Lo más habitual, según algunos autores (Pitt, 1973), es que durante los diez primeros días tras el parto se produzca la denominada tristeza postparto, maternity blues o baby blues. En este sentido, muchas mujeres se sienten especialmente culpables por tener sentimientos depresivos cuando creen que deberían estar contentas y puede darse el caso de que sean reticentes a manifestar sus síntomas o sus sentimientos negativos hacia el niño. Victoroff comparó este estado emocional con el síndrome premenstrual ya que entre las particularidades que le caracterizan destacan las siguientes: labilidad emocional acompañada de llanto, irritabilidad, ansiedad, hipocondriasis e insomnio en los 10 días siguientes al nacimiento del bebé (Pritchard y Harris, 1996).

Con posterioridad, la situación puede complicarse si persisten los síntomas después de esos diez primeros días. Así, según la Asociación Psiquiátrica Americana (APA, 1994), es frecuente que las mujeres con episodios depresivos mayores postparto presenten una gran ansiedad, crisis de angustia, desinterés por el nuevo hijo e insomnio, llanto espontáneo, etc. mucho después de lo que suele durar la tristeza postparto pasajera. Esta depresión más grave, coincide con lo que Pitt (1968) describió como depresión atípica y cuya duración se puede prolongar durante varios meses. La sintomatología que señaló Pitt en sus investigaciones es semejante a la descrita por la APA destacando las siguientes características: fatiga, irritabilidad hacia el marido y otros niños, ansiedad específica sobre el bebé, falta de apetito, insomnio, falta de interés por el sexo y desmoralización.

Por último señalamos los estados de psicosis puerperal que en sus formas más graves pueden implicar un serio peligro para las vidas de la madre y /o del niño. Suelen comenzar en las seis a doce semanas siguientes al parto y se caracterizan por presentar una sintomatología desigual a lo largo del tiempo incluyendo alucinaciones, delirios y estados de confusión (Pritchard y Harris, 1996; Terp y Mortensen, 1998). En este sentido, la APA (1994) señala, dentro de los trastornos del estado de ánimo, el apartado Especificación de inicio en el postparto donde se pueden incluir tanto la existencia de un episodio depresivo mayor, maníaco o mixto, un trastorno depresivo mayor, un trastorno bipolar I o II o un trastorno psicótico breve, dependiendo de los criterios diagnósticos que se cumplan.

Si bien ha existido un prolongado debate respecto a la consideración de esta diversa sintomatología como alteraciones que indican la existencia de un trastorno con entidad propia, hoy día, tanto en la clasificación de la APA ya indicada (APA, 1994) como en la Clasificación Internacional de Enfermedades (CIE-10) se incluye un apartado específico que da cuenta de su evidente importancia: Tras- 
tornos mentales y del comportamiento en el puerperio, no clasificados en otro lugar (F53) (O.M.S., 1992).

Así pues, los trastornos del estado de ánimo durante el puerperio conllevan una serie de consecuencias para la madre donde se ven mermados tanto su nivel de salud como la capacidad para experimentar el gozo de la maternidad. Incluso se ha llegado a establecer que a pesar de que existen unas tasas bajas de suicidio de mujeres en el periodo postnatal, aquellas que desarrollan una depresión grave, sobre todo en el primer año tras el parto, están en un riesgo elevado de cometer suicidio (Appleby, Mortensen y Faragher, 1998)

Esta sintomatología afecta de forma negativa al desarrollo de los hijos y contribuye también al deterioro progresivo de las relaciones conyugales y familiares. Dadas estas premisas es evidente que la maternidad es un cambio en la vida de la mujer que influye no sólo en su cuerpo sino en sus relaciones sociales, su identidad, su conducta y sus futuros proyectos de vida.

\section{ETIOLOGÍA DE LA DEPRESIÓN POSTPARTO}

Uno de los aspectos más controvertidos del estudio de la depresión postparto es su etiología. A pesar de los numerosos factores causales que se han planteado a lo largo de diversas investigaciones, no se ha encontrado ninguno cuya contribución específica y patognomónica se haya demostrado claramente. Entre tales agentes causales se puede citar el hecho de que la mujer sea multípara (Davidson, 1972), dificultades para el amamantamiento y/o preocupaciones sobre el bebé así como alteraciones hormonales (Pitt, 1973), falta de apoyo social para la mujer (Lane, Keville, Morris, Kinsella, Turner y Barry, 1997; Paykel, Emms, Fletcher y
Rassaby, 1980), existencia de problemas entre la pareja (Tod y Edin, 1964), autoeficacia parental (Maciejewski, Prigerson y Mazure, 2000), entre otras.

Tradicionalmente, en la etiología y patogenia de los trastornos mentales que sobrevienen en el puerperio se han citado la existencia de infecciones, las pérdidas de sangre ya sean bruscas o repetidas, errores de dietética y/o empleo de drogas anorexígenas durante el embarazo, experiencia psicológica del embarazo y del parto (Porot, 1977). Evidentemente, aunque se ha evolucionado en el control de los factores más básicos tales como las infecciones o las hemorragias es cierto que podemos encontrar todavía algunos de los problemas señalados, sobre todo los que hacen referencia a problemas de dietética (Logue, 1991).

Pritchard y Harris (1996) revisando la literatura especializada en alteraciones psiquiátricas perinatales especifican diversos mecanismos etiológicos. Respecto a la tristeza postparto o maternity blues señalan la importancia de diversos factores sociales y biológicos destacando como posibles contribuciones el cambio en el nivel de progesterona, la historia de síndrome premenstrual, dificultades paternas, pobre ajuste durante el embarazo y existencia previa de depresión. Al estudiar la depresión postnatal, los autores inciden en la mayor claridad de factores asociados entre los que se encuentran la edad, el apoyo social, los eventos estresantes y la existencia previa de enfermedad mental. Con referencia a la edad, tanto las mujeres más jóvenes como las de más edad tienen un mayor riesgo de presentar depresión postparto. Aquellas mujeres con una pareja que no ofrece apoyo, ya sea emocional o práctico, también presentan un riesgo mayor. En cuanto a los acontecimientos estresantes, se ha encontrado que en el año anterior al parto la muestra que presentaba depresión postparto había experimen- 
tado el doble de eventos que el grupo control.

En último lugar, al igual que en los análisis centrados en la maternity blues, y tal como se esperaba, la presencia previa de enfermedad mental es otro factor de riesgo importante.

Llavona, Muruaga y Nogueiras (1986) plantean desde la perspectiva operante un modelo explicativo que integra diversos elementos que se habían puesto de manifiesto en anteriores estudios. Señalan que la depresión postparto "puede considerarse como el resultado de un proceso fundamentalmente operante que se produce después del parto, donde la mujer se encuentra con un organismo debilitado, por el esfuerzo que supone el parto y por el reajuste fisiológico posterior, y con unos cambios hormonales que quizá faciliten la inestabilidad emocional" (p.206). Al respecto, destacan el papel de las consecuencias que se deriven de la conducta del cuidado del hijo. La madre, tras el parto, presenta un exceso de actividad que incrementa el estado de fatiga, alteraciones en los patrones de sueño, un estado de alerta constante para cuidar al bebé y, evidentemente, como ya se ha señalado, se ve influida por factores fisiológicos importantes que también modulan su comportamiento. Además de esta situación, las relaciones interpersonales, sexuales y el ocio pueden haber sufrido cambios importantes. En esta situación pueden existir circunstancias que se vivan como consecuencias puramente aversivas que van a castigar la conducta de la madre en lugar de reforzarla, tales circunstancias pueden ser, por ejemplo, que el niño tenga problemas de salud o de alimentación, o que el funcionamiento del niño no se corresponda con el comportamiento de la madre, que la pareja presente discrepancias en el cuidado de los hijos que a su vez conllevan discusiones, o que la pareja se sienta relegada a un segundo plano, etc. Si por el contrario, las consecuencias son reforzantes, tanto por parte del hijo como de la pareja, se compensará la presencia de estimulación aversiva proveniente de su organismo y la madre seguirá manteniendo la conducta de cuidado sin sufrir la depresión.

\section{PREVALENCIA}

La prevalencia de la depresión postparto es difícil de establecer. Los datos oscilan según las investigaciones, desde un $5.8 \%$ (Harris, Lovett, Smith, Read, Walker y Newcombe, 1996), un 8.8\% (Alvarado, Vera, Rojas, Olea, Monardes y Neves,1992) un $9.2 \%$ (Jadresic, Jara, Miranda, Arrau y Araya, 1992) o un $13.5 \%$ (Pedros, Tomas, Gómez, Leal y García, 1998) hasta un 18\% (Stuart, Couser, Schilder, O'Hara y Gorman ,1998) e incluso en torno al $50 \%$ según otros investigadores (Bagedahl y Ruppert, 1998; Manzano, Righetti-Veltema y Conne-Perréard,1997). Esta disparidad en las cifras señaladas puede estar relacionada con la complejidad conceptual del síndrome denominado "depresión postparto" ya que, en muchos artículos, no queda patente si se está haciendo referencia a una tristeza pasajera -maternity blues- a una depresión más duradera y grave o incluso a una psicosis puerperal. Por otra parte, también hemos de señalar como posibles elementos causales de estas diferencias la diversidad de instrumentos utilizados para diagnosticar este trastorno así como la falta de análisis longitudinales, hecho que puede sesgar los datos según el tiempo transcurrido desde el momento del parto y la realización del estudio.

\section{OBJETIVO DEL PRESENTE ESTUDIO}

Las consecuencias de las alteraciones emocionales perinatales son costosas no sólo en cuanto a sufrimiento personal 
sino también respecto a su potencial de incremento de demandas de los servicios de salud.

Nuestro objetivo general consistió por tanto en analizar la existencia de depresión postparto en una muestra de mujeres de nuestra Comunidad y confirmar al cabo de aproximadamente dos meses, si persistían las alteraciones. Como objetivo a largo plazo y más específico, se pretende que la presente investigación sirva para contribuir a paliar la sintomatología depresiva en aquellas mujeres que la presenten en el momento inmediato al parto, colaborando por tanto en la prevención de las complicaciones posibles del trastorno en futuras ocasiones gracias al diagnóstico precoz del mismo. De esta forma se favorece que los trastornos mentales existentes sean reactivos, transitorios y benignos, repercutiendo como consecuencia, en una mejora de la eficiencia del sistema sanitario y, en definitiva, en una mejora de la sociedad.

\section{METODOLOGÍA}

\section{Participantes}

La muestra estuvo formada por un grupo de 101 mujeres residentes en la Ciudad de Valencia y pueblos de alrededor que dieron a luz en el Centro Maternal del Hospital Universitario «La Fe» de Valencia. Una enfermera y psicóloga recogió la información de las variables clínicas y sociodemográficas, la anotó en una hojaregistro y comprobó que las mujeres cumplimentaran los instrumentos los primeros días tras el parto. Se incluyeron en la muestra todas las mujeres cuyo alumbramiento coincidió con el turno laboral de la enfermera, no existiendo aleatorización ni selección previa. Un número elevado de mujeres eran primíparas (65\%), algunas tenían ya un hijo (22\%), otras dos $(7 \%)$ tres $(3 \%)$ e incluso cuatro $(3 \%)$. La edad media era de aproximadamente 30 años, abarcando un rango de 18 a 42 años $(X=29.67 ; D T=4.48)$. La mayoría de ellas estaban casadas ( $84 \%$ ) e informaron pertenecer socioeconómicamente a clase media $(79 \%)$. La mayoría de las mujeres tenía estudios primarios (55\%) seguido por un número mucho menor que poseía formación profesional (17\%), estudios universitarios (12\%), COU (12\%) y Bachiller $(4 \%)$. En cuanto a la profesión, un porcentaje bastante elevado de mujeres eran amas de casa ( $47 \%$ ), seguido por un número considerable que tenían un trabajo cualificado tales como administrativas, docentes, etc. $(30 \%)$, trabajadoras manuales $(22 \%)$ y en paro $(1 \%)$.

\section{Instrumentos de evaluación y procedimiento}

De los instrumentos utilizados tradicionalmente para evaluar la existencia de alteraciones mentales en el postparto, seleccionamos los siguientes: La Escala de Edimburgo para la Depresión Postparto (EPDS), el Inventario de Depresión de Beck (BDI) y el Inventario de Ansiedad Estado-Rasgo (STAI). Los tres instrumentos se administraron tras el parto y aproximadamente a los dos meses del mismo, con el fin de examinar si existían cambios a lo largo del tiempo. Se incluyó también una hoja de recogida de datos sociodemográficos y clínicos con el propósito de explorar otras variables clínicas que habían mostrado su relevancia en la literatura especializada sobre el tema. Dichas variables clínicas incluían datos acerca del embarazo y del parto, hospitalización del hijo recién nacido, atención hospitalaria recibida, y diagnóstico previo de depresión.

- Escala de Edimburgo para la Depresión Postparto (Edimburg Postnatal Depression Scale, EPDS) de Cox, Holden y 
Sagovsky (1987). Puesto que el objeto de análisis era examinar la sintomatología depresiva en el postparto se utilizó este cuestionario, específicamente diseñado para tal fin. Las diversas publicaciones centradas en la validación del EPDS en distintas poblaciones y culturas muestran valores elevados tanto en sensibilidad como en especificidad para evaluar la depresión postparto en mujeres (Areias, Kumar, Barros y Figueiredo, 1996; Ghubash y Abou-saleh, 1997). Es un instrumento de fácil administración que puede cumplimentarse en tan sólo unos minutos. Consta de diez items con cuatro alternativas de respuesta (rango de 0 a 3) y existe una versión en español traducida y validada por Jadresic, Araya y Jara (1995) en población chilena, donde se confirman sus buenas cualidades psicométricas, respecto a los índices de consistencia interna $(\mathrm{a}=$ 0.77) y de validez externa comparada con los criterios de clasificación de Spitzer, Endicott y Robins (Research Diagnostic Criteria RDC, 1978). Los puntos de corte clínicos para diferenciar entre mujeres con y sin depresión post-parto se han establecido en el umbral de 9/10 y reflejan una sensibilidad del $100 \%$ y una especificidad del $80 \%$.

- Inventario de Depresión de Beck (Beck Depression Inventory, BDI, Beck, Rush, Shaw y Emery, 1979). Es una de las herramientas de evaluación más utilizadas para cuantificar los síntomas depresivos así como su intensidad en poblaciones normales y clínicas y presenta buenas propiedades psicométricas en los distintos trabajos publicados tanto en la versión original como en la traducida al castellano (Beck, Steer y Garbin, 1988; Vázquez y Sanz, 1997, 1998). Con referencia al uso de este inventario, somos conscientes de la superioridad que muestra el EPDS en el periodo del postparto en determinados estudios al compararlos con el BDI (Pritchard y Harris, 1996), pero destacamos que en dichas investigaciones se ha utilizado la versión de 1961. Nosotros hemos preferido la versión posterior de 1978 ya que presenta evidentes ventajas frente a la anterior (Vázquez y Sanz, 1997, 1998), entre las que destaca la inclusión del enunciado «durante la última semana» aludiendo a la duración de la sintomatología y que se acerca más a los criterios diagnósticos planteados por la APA (1994). Se ha escogido un punto de corte clínico igual a 18 ya que en numerosas ocasiones se ha puesto de manifiesto que dicha puntuación maximiza la exactitud diagnóstica en cuanto a sensibilidad, especificidad, poder de predicción y eficiencia diagnóstica y es una puntuación que goza del consenso de los investigadores para diferenciar entre personas con y sin depresión (Vázquez y Sanz, 1997).

- Inventario de Ansiedad Rasgo-Estado (State-Trait Anxiety Inventory, STAI) de Spielberger, Gorsuch y Lushene (1970) cuyo objetivo es medir el nivel de ansiedad de los sujetos. En la presente investigación se ha utilizado la versión editada por TEA en 1997 ( $4^{\mathrm{a}}$ ed.) aunque únicamente se ha incluido la escala de ansiedad-estado con el fin de analizar la existencia de cambios en el estado de ansiedad de esta muestra.

Se realizó un primer pase de todos los instrumentos señalados anteriormente (EPDS, BDI, STAI) para evaluar la existencia de sintomatología depresiva y ansiosa en la muestra seleccionada. Posteriormente, tal como se ha indicado previamente y con el objetivo de comprobar si se trataba de alteraciones emocionales transitorias o no, se administraron nuevamente los cuestionarios mencionados al cabo de aproximadamente dos meses. 
En todos los casos fueron las propias mujeres las que cumplimentaron los cuestionarios puesto que el formato era de autoinforme. En aquellas situaciones en que hubo necesidad de mayor explicación de algún ítem por falta de comprensión del mismo, se procuró no sesgar la información. Para ello, se contó con la colaboración de una psicóloga que supervisó la cumplimentación de los instrumentos en los distintos momentos del estudio.

\section{RESULTADOS}

\section{a) Variables Clínicas}

Uno de los aspectos que hemos investigado es la vivencia y experiencia del embarazo. Las mujeres que componen la muestra señalaron en su mayoría ( $85 \%$ ) sentirse muy contentas o contentas $(1 \%)$ al conocer que estaban esperando un hijo, si bien un porcentaje relativamente importante se sentía muy contrariada $(10 \%)$ o algo contrariada (4\%). En cuanto al embarazo como experiencia vital, para un elevado porcentaje de mujeres fue una experiencia positiva, siendo excelente para la mayoría de ellas (61\%) $y$ buena para otras $(12 \%)$, aunque un tercio de la muestra experimentó una experiencia "regular» $(25 \%)$, y sólo un pequeño número de mujeres indicó una experiencia negativa: "mala» $(1 \%)$ y "pésima» (1\%).

Otra variable observada, la experiencia del parto no parece mostrar resultados tan alentadores como los anteriores. Un porcentaje bastante elevado de mujeres señaló como "regular» dicha experiencia (53\%) seguido de un porcentaje algo menor de mujeres que sí la señalaron como "excelente» (30\%), y el resto indicó que fue una experiencia "pésima» $(8 \%)$, «buena» (5\%), "mala» (4\%). En este sentido, es relevante indicar los dis- tintos tipos de parto ya que si bien la mayoría de las mujeres tuvo un parto vaginal normal (68\%), un número considerable de mujeres fueron sometidas a cesárea $(24 \%)$ y en un porcentaje menor de la muestra fue necesario un parto instrumentado ( $8 \%)$. No obstante, la anestesia epidural se utilizó en muy pocas mujeres $(11 \%)$ si bien recientemente se ha incrementado su uso, dato que puede tener un papel destacado en los resultados obtenidos.

La mayoría de las mujeres a las que se les preguntó sobre su percepción de capacidad para enfrentarse a esa situación posterior al parto, señaló que iban a ser capaces de ello, oscilando entre sentirse totalmente capaces ( $73 \%)$, bastante capaces $(8 \%)$ o normales $(18 \%)$. De hecho, no hubo ninguna mujer que señalara que se sentía incapaz de afrontar los cambios en su vida ante el nacimiento de su hijo/a, y sólo un $1 \%$ se sentía poco capaz.

Se recabaron datos acerca de la hospitalización del hijo encontrándose un número importante de mujeres en las que hubo necesidad de ingresar al recién nacido. Así, un $53 \%$ de los sujetos no tenía el hijo/a hospitalizado frente al $47 \%$ que sí lo tenía siendo la causa principal la prematuriedad y/o bajo peso al nacer $(72 \%)$.

Un elevado porcentaje de mujeres tenía previsto dar lactancia materna $(71 \%)$ y de ellas la mayoría $(70 \%)$ lo iba a hacer durante más de dos meses.

Una variable señalada como positiva era la atención hospitalaria recibida. La mayoría de las mujeres señaló que la atención recibida fue excelente $(76 \%)$ o buena $(7 \%)$ si bien el resto de la muestra no consideró positiva dicha atención $(17 \%)$, oscilando de regular (13\%) a peor (3\%) y pésima ( $1 \%)$.

La última variable recogida fue la existencia previa de diagnóstico de depresión. La mayoría de las mujeres que com- 
ponen la muestra, nunca habían sufrido de depresión (81\%). Así, un 19\% «informan" de haber sido diagnosticadas previamente de depresión, siendo semejantes estos valores a la prevalencia del trastorno según cita la APA (1994). Lamentablemente, no se ha podido especificar el tipo de depresión y sólo se ha podido confirmar la existencia de tratamiento psiquiátrico-farmacológico solo o combinado con tratamiento psicológico en algunas de ellas $(n=12)$ ya que el resto de las mujeres informó que el tratamiento lo pautó el médico de cabecera $(n=4)$ e incluso algunas de ellas comentó «haber salido de la depresión" por sí sola o con ayuda de la pareja $(n=2)$, sólo una mujer recibió tratamiento psicológico sin apoyo farmacológico $(n=1)$. El tiempo transcurrido desde dicho diagnóstico es diferente en cada caso aunque en la mayoría la depresión había ocurrido tres años o más antes del último parto $(n=16)$, una mujer estuvo en tratamiento psicológico en los seis primeros meses del embarazo de su único hijo y dos mujeres estuvieron en tratamiento un año antes del parto. En la actualidad, ninguna de ellas estaba recibiendo tratamiento ni farmacológico ni psicológico.

\section{b) Cuestionarios}

En primer lugar, las puntuaciones medias de la muestra no fueron elevadas en ninguno de los dos momentos evaluados y, en segundo lugar, resaltamos el hecho de que las puntuaciones en depresión evolucionan de forma diferente dependiendo del tipo de cuestionario utilizado (EPDS o BDI) tal como puede observarse en la Tabla 1. Así, en cuanto a la evolución de las puntuaciones, se puede observar que según los datos ofrecidos tras la administración del BDI la puntuación media aumenta en el segundo pase, mientras que con el uso del
EPDS la puntuación en depresión es menor al cabo de dos meses. Sin embargo, hemos de señalar que mientras que el aumento de depresión que se observa con el BDI. no es significativo, la disminución de depresión con el EPDS si resultó ser significativa.

$\mathrm{Si}$ nos atenemos estrictamente a la existencia de depresión en la muestra según el punto de corte de cada cuestionario se observa que un porcentaje considerable de mujeres supera el punto de corte establecido por Jadresic, Araya y Jara (1995) (EPDS >9) tanto en el momento más cercano al parto $(32.67 \%)$ como posteriormente $(26.73 \%)$. Respecto al BDI, y teniendo también en cuenta el punto de corte establecido (18), sólo existe un porcentaje de mujeres que lo supera en el primer pase ( $4.95 \%)$, ya que en la segunda administración del instrumento la puntuación máxima observada es de 12 .

En el caso del STAI-E, hay que señalar que las puntuaciones medias de la muestra no parecen ser elevadas en ninguno de los dos pases. Además, se observa (véase también la Tabla 1) cómo la ansiedad, en su vertiente situacional, disminuye de forma significativa con el paso del tiempo en las mujeres de la presente muestra.

Si se tienen en cuenta los percentiles que corresponden a las puntuaciones directas obtenidas tras la aplicación del cuestionario, parece interesante destacar que, en general, a la mayoría de las mujeres les corresponden centiles bastante bajos en la variable ansiedad. Así, el $48.5 \%$ de las mujeres del primer pase se incluyen en percentiles de 25 o inferiores, porcentaje que se eleva al cabo de dos meses, pues el $75 \%$ de las mujeres presentan percentiles iguales o menores a 25. Se observa, pues, una disminución importante y significativa en cuanto a la ansiedad estado en las mujeres que componen la presente muestra. 
Tabla 1. Comparación de medias de las variables de depresión y ansiedad a través de los cuestionarios BDI, STAI-E y EPDS, en los dos momentos del estudio

\begin{tabular}{|c|c|c|c|c|c|}
\hline & \multicolumn{2}{|c|}{ Primer momento } & \multicolumn{2}{|c|}{ Segundo momento } & $t$ \\
\hline BDI & 5,75 & 5,05 & 6,41 & 2,08 & $-1,40$ \\
\hline EPDS & 8,17 & 4,98 & 7,36 & 3,18 & $2,92^{\star}$ \\
\hline STAI-E & 17,39 & 10,37 & 12,64 & 4,54 & $4,76^{\star \star}$ \\
\hline
\end{tabular}

Nota: * $p \leq 0,01 ;{ }^{*}{ }^{\star} p \leq 0,001$; BDI (Beck Depression Inventory); EPDS (Edimburgh Postnatal Depression Scale); STAI-E (State-Trait Anxiety Inventory)

\section{c) Relaciones entre variables clínicas y cuestionarios}

Por último, al analizar las variables clínicas y su relación con la variable de depresión, hemos obtenido los resultados que se comentan a continuación.

Ya se ha señalado anteriormente cómo el tener o no un hijo hospitalizado se había incluido como variable clínica a tener en cuenta ya que podía suponer que si una madre tenía a su hijo hospitalizado, puntuaría más en depresión que si no lo tenía, aunque en la mayoría de los casos dicha hospitalización era debida simplemente a prematuriedad y/o bajo peso del recién nacido. El presente estudio nos indica que, efectivamente, las mujeres cuyo hijo recién nacido estaba hospitalizado en el momento de la evaluación, puntuaban más en depresión evaluada a través de EPDS en el primer pase $(t=-2,03 ; p \leq 0,05)$.

En segundo lugar, tanto la experiencia del embarazo como el sentirse capaz o no de afrontar los cambios tras el parto son variables que han demostrado su importancia en relación con la presencia o no de depresión.

Así, las mujeres que consideraban una experiencia regular o pésima del embarazo puntuaban más en depresión que quienes lo habían experimentado positivamente tanto en el primer pase $(t=-2,96$; $p \leq 0,01)$ como en el segundo $(t=-2,67 ; p \leq$ $0,05)$, según el EPDS Relación que se ratifica utilizando e[l BDI pero sólo en la pri- mera administración del instrumento $(t=$ $-2,01 ; p \leq 0,05)$. Por otra parte, aquellas mujeres que se sentían poco capaces de afrontar los cambios venideros en el postparto (recordemos que no había ninguna que se sintiera incapaz) puntuaban más en depresión evaluada a través del EPDS tanto en el primer pase $(t=-2,74$; $p \leq 0,05)$ como en el segundo $(t=-2,66 ; p \leq$ $0,05)$.

Por último, en cuanto a la experiencia o diagnóstico previo de depresión, en la presente investigación se confirma nuevamente la importancia de esta variable, ya que las diferencias encontradas son significativas. Las mujeres que habían sido diagnosticadas de depresión anteriormente, puntúan más en depresión cuando ésta es evaluada a través del BDI ( $t=-2,75 ; p \leq 0,05$ en el primer pase) o a través del EPDS en ambos pases: $(t=-$ $2,48 ; p \leq 0,05)$ en el primer pase, $(t=-3,28$; $p \leq 0,01$ ) en el segundo pase.

Por otra parte y con referencia a las variables clínicas relevantes y la ansiedad, se destaca únicamente la variable de «sentirse poco capaces para afrontar los cambios". Las mujeres que habían señalado que se sentían poco capaces para afrontar los cambios que ocurrían en el postparto, obtenían puntuaciones más elevadas en ansiedad estado que las mujeres que se sentían capaces o muy capaces $(t=-2,32 ; p \leq 0,05)$ siendo esa diferencia significativa únicamente en la primera evaluación, pero no transcurridos los dos meses. 
En cuanto a los resultados obtenidos con el análisis correlacional entre las variables sociodemográficas y clínicas y las puntuaciones en depresión y ansiedad, destacamos lo siguiente (ver Tabla 2)

Una de las variables que muestra un mayor numero de correlaciones es la experiencia del embarazo que ha demostrado su importancia respecto a la experiencia del parto, a la capacidad para afrontar cambios y en cuanto a la depresión evaluada a través de los dos instrumentos utilizados (BDI y EPDS).

En segundo lugar, la capacidad percibida por las mujeres para afrontar los nuevos cambios también es una variable clínica que muestra una relación significativa y negativa con depresión aunque sólo si ésta es evaluada a través del EPDS.

Por otra parte resalta el hecho de que, la edad, una variable mencionada en muchos estudios, no parece ser relevante en la presente investigación, pero sí el número de hijos ya que muestra una relación negativa significativa al evaluar la depresión con el EPDS.

Existen otras relaciones no menos importantes aunque no se refieran estrictamente a las variables de depresión y/o ansiedad. Es de destacar que la atención hospitalaria, por ejemplo, muestra correlaciones notables con la experiencia del parto y con la capacidad percibida para afrontar nuevos cambios. Es hasta cierto punto obvio, que únicamente se dé tal relación en la primera administración del instrumento pues era el momento en que las mujeres estaban ingresadas en el servicio de obstetricia del Centro Maternal del Hospital Universitario La Fe de Valencia.

\section{DISCUSIÓN}

El objetivo de la presente investigación era analizar la existencia de depresión postparto en un grupo de mujeres de nuestra Comunidad y comprobar si ésta persistía al cabo de dos meses. Se ha podido observar que un porcentaje numeroso de mujeres supera los puntos de corte establecidos para el diagnóstico de depresión según la escala EPDS, en ambos momentos del estudio.

No obstante, creemos que los resultados obtenidos en la primera administración del instrumento indican más bien disforia, en el sentido de la "maternity blues» que ocurre en los primeros días tras el parto y que ya señalara Pitt en sus investigaciones (1973). Sin embargo, el porcentaje observado en los resultados del segundo pase $(26,73 \%)$ sigue siendo elevado y se incluye en el periodo de tiempo señalado por otros investigadores donde el EPDS se muestra como un instrumento sensible para detectar depresion postnatal (Bergant, Heim, Hulmer e Illmensee, 1999).

Tabla 2. Correlaciones significativas entre las variables sociodemográficas y clínicas y las variables de depresión y ansiedad en los dos momentos del estudio (datos del segundo momento, entre paréntesis)

\begin{tabular}{|c|c|c|c|c|}
\hline & $\begin{array}{c}\text { Experiencia } \\
\text { parto }\end{array}$ & $\begin{array}{c}\text { Capaz afrontar } \\
\text { cambios }\end{array}$ & $B D I$ & $E P D S$ \\
\hline $\begin{array}{l}\text { Experiencia embarazo } \\
\text { Capaz afrontar cambios } \\
\mathrm{N}^{\mathrm{Q}} \text { hijos } \\
\text { Atención hospitalaria }\end{array}$ & $\begin{array}{l}0,27^{\star} \\
\text { n.s. } \\
\text { n.s. } \\
0,24+\end{array}$ & $\begin{array}{c}0,22+ \\
\text { n.s. } \\
\text { n.s. } \\
0,28^{\star}\end{array}$ & $\begin{array}{l}-0,20+ \\
\text { n.s. } \\
\text { n.s. } \\
\text { n.s. }\end{array}$ & $\begin{array}{c}-0,33+(-0,26)^{\star} \\
-0,25+ \\
-0,20+ \\
\text { n.s. }\end{array}$ \\
\hline
\end{tabular}

Nota: $+p \leq 0,05 ;{ }^{\star} p \leq 0,01$; BDI (Beck Depression Inventory); EPDS (Edimburgh Postnatal Depression Scale). 
Las puntuaciones en depresión muestran una disminución significativa con el paso del tiempo, disminución que también han indicado otros autores (Stuart et al., 1998) y que no se ha corroborado en otros estudios ya que el porcentaje de mujeres que tenía depresión era similar en los dos períodos estudiados (Lane et al., 1997).

Respecto al instrumento mencionado, sería conveniente adaptarlo a la población española estableciendo puntos de corte diferenciales que sirvieran para determinar diversos tipos de trastorno depresivo tal como se ha establecido en otras poblaciones como la anglosajona (Murray y Cox, 1990) o la portuguesa (Areias et al., 1996), ya que las mujeres que desarrollan una depresión grave en el primer año tras el parto tienen un riesgo elevado de cometer suicidio y muestran un deterioro progresivo de las relaciones familiares y conyugales (Appleby, Mortensen y Faragher, 1998).

Si nos centramos en las variables clínicas analizadas, señalamos la importancia de la experiencia del embarazo, la capacidad para afrontar cambios y el diagnóstico previo de depresión. En este sentido, sería apropiado profundizar en estas variables teniendo en cuenta la posible relación causal con el tipo de parto (instrumentado, cesárea, etc.) o las estrategias de afrontamiento utilizadas (ya sean cognitivas o conductuales), el apoyo social o la existencia de eventos estresantes en el año previo al parto. También indicamos la conveniencia de volver a analizar la experiencia del parto ya que en el último año se ha incrementado el uso de la anestesia epidural en el Hospital que colaboró para la obtención de la muestra.

\section{REFERENCIAS BIBLIOGRÁFICAS}

APA (1994). Diagnostic and Statistical Manual of Mental Disorders, $4^{\text {th }}$ Edition. DSM-IV. Washington, D.C.: American Psychiatric Association.

Alvarado, R., Vera, A., Rojas, M., Olea, E, Monardes, J., y Neves, E. (1992). La Escala de Edimburgo para la detección de cuadros depresivos en el post-parto. Revista de Psiquiatría, 3-4, 1177-81

Appleby, L., Mortensen, P.B., y Faragher, E.B. (1998). Suicide and other causes of mortality after post-partum psychiatric admission. British Journal of Psychiatry, 173, 209-211.

Areias, M.E.G., Kumar, R., Barros, H., y Figueiredo, E. (1996). Comparative incidence of depression in women and men, during pregnancy and after childbirth. Validation of the Edinburgh Postnatal Depression Scale in Portuguese Mothers. British Journal of Psychiatry, 169, 30-35.

Bagedahl, S.M., y Ruppert, S. (1998). Parapartum mental illness: A long-term followup study. Psychopathology, 31, 250-259.

Beck, A.T., Rush, A.J., Shaw, B.F., y Emery, G. (1979). Cognitive therapy of depression. New York: Guilford Press (Traducción española en Bilbao: Descleé de Brouwer, 1983)

Beck, A.T., Steer, R.A., y Garbin, M.C. (1988). Psychometric properties of the Beck Depression Inventory: Twenty-five years of evaluation. Clinical Psychology Review, 8, 77-100.

Bergant, A., Heim, K., Hulmer, H., e Illmensee, K. (1999). Early postnatal depressive mood: associations with obstetric and psychosocial factors. Journal of Psychosomatic Research, 46, 391-394.

Cox, J.L., Holden, J.M., y Sagovsky, R. (1987). Detection of postnatal depression: Development of the 10-item Edinburgh Postnatal Depression Scale. British Journal of Psychiatry, 150, 782-786.

Davidson, J.R. (1972). Postpartum mood change in Jamaican women: A description and discussion on its significance. British Journal of Psychiatry, 121, 659-663.

Ghubash, R., y Abou-Saleh, M.T. (1997). Postpartum psychiatry illness in Arab culture: prevalence and psychosocial correlates. 
British Journal of Psychiatry, 171, 65-68. Harris, B., Lovett, L., Smith, J., Read, G., Walker, R., y Newcombe, R. (1996). Cardiff puerperal mood and hormone study. III. Postnatal depression at 5 to 6 weeks postpartum, and its hormonal correlates across the peripartum period. British Journal of Psychiatry, 168, 739-744.

Jadresic, E., Araya, R., y Jara, C. (1995): Validation of the Edinburgh Postnatal Depression Scale (EPDS) in Chilean postpartum women. Journal of Psychosomatic Obstetrics Gynecology, 16, 187-191.

Jadresic, E., Jara, C., Miranda, M., Arrau, B., y Araya, R. (1992): Trastornos emocionales en el embarazo y el puerperio: estudio prospectivo de 108 mujeres. Revista Chilena de Neuropsiquiatría, 30, 99-106.

Lane, A., Keville, R., Morris, M., Kinsella, A., Turner, M., y Barry, S. (1997). Postnatal depression and elation among mothers and their partners: prevalence and predictors. British Journal of Psychiatry, 171, 550-555.

Logue, A.W. (1991). The psychology of eating and drinking: An introduction. New York: Freeman \& Co., Publishers.

Llavona, L.M., Muruaga, S., y Nogueiras, B. (1986). Enfoque conductual de la depresión postparto. Revista Española de Terapia del Comportamiento, 4, 193-220.

Maciejewski, P. K., Prigerson, H.G., y Mazure, C.M. (2000). Self-efficacy as a mediator between stressful life events and depressive symptoms. British Journal of Psychiatry, 176, 373-378.

Manzano, J., Righetti-Veltema, M., y ConnePerréard, E.C. (1997). Le syndrome de depression du pre-partum. Resultat d'une recherche sur les signes recurseurs de la depression du post-partum. Psychiatrie de l'Enfant, 40, 533-552.

Murray, D., y Cox, J. (1990). Screening for postnatal depression during pregnancy with the Edinburgh Postnatal Depression Scale (EPDS). Journal of Reproductive and Infant Psychology, 8, 99-107.

Organización Mundial de la Salud (1992). CIE-10. Trastornos mentales y del comportamiento. Descripciones clínicas y pautas para el diagnóstico. Edición en español. Madrid: Meditor.
Paykel, E.S., Emms, E.M., Fletcher, J., y Rassaby, E.S. (1980). Life events and social support in puerperal depression. British Journal of Psychiatry, 130, 339-346.

Pedros, A., Tomas, A., Gómez, M., Leal, C., y García, K (1998). Estilo de crianza en madres con depresión postparto, Actas Luso Españolas de Neurología, Psiquiatría y Ciencias Afines, 26, 104-110.

Pitt, B. (1968). Atypical depression following childbirth. British Journal of Psychiatry, 114, 1325-1335.

Pitt, B. (1973). Maternity Blues. British Journal of Psychiatry, 122, 431-433

Porot, A. (1977). Diccionario de Psiquiatría. Clínica y Terapéutica. Tomo II. 1001-1004. Barcelona: Editorial Labor.

Pritchard, D.B., y Harris, B. (1996). Aspects of perinatal psychiatric Illness. British Journal of Psychiatry, 169, 555-562.

Spielberger, C.D., Gorsuch, R.L., y Lushene, R.E. (1970). STAI, Manual for the State-Trait Anxiety Inventory (Self Evaluation Questionnaire). Palo Alto, California, USA: Consulting Psychologists Press. (Adaptación española: Cuestionario de Ansiedad EstadoRasgo. Madrid: TEA Ediciones S.A., 1997)

Spitzer, R.L., Endicott, J., y Robins, E. (1978). Research Diagnostic Criteria (RDC) for selected group of functional diagnosis. Nueva York: State Psychiatric Institute.

Stuart, S., Couser, G., Schilder, K., O'Hara, M., y Gorman, L. (1998): Postpartum anxiety and depression: Onset and comorbidity in a community sample. Journal of Nervous and Mental Disease, 186, 420-424.

Terp, I.M., y Mortensen, P.B. (1998). Post-partum psychoses. Clinical diagnoses and relative risk of admission after parturition. British Journal of Psychiatry, 172, 521-526.

Tod, E.D., y Edin, M.B. (1964). Puerperal depression: a prospective epidemiological study, Lancet, 2, 1264-1266.

Vázquez, C., y Sanz, J. (1998). Fiabilidad y validez de la versión española del Inventario para la Depresión de Beck de 1978 en pacientes con trastornos psicológicos. Clínica y Salud, 10, 59-81.

Vázquez, C., y Sanz, J. (1997). Fiabilidad y valores normativos de la versión española del inventario para la depresión de Beck de 1978. Clinica y Salud, 8, 403-422. 